\title{
Sintomas de Ansiedade, Depressivos e Uso de Substâncias Psicoativas em Pacientes Após a Cirurgia Bariátrica
}

\author{
Symptoms of Anxiety, Depressive and Psychoactive Substance use in Patients After \\ Bariatric Surgery
}

\section{Síntomas de Ansiedad, Depresivos y Uso de Sustancias Psicoactivas en Pacientes Después de Cirugía Bariátrica}

\author{
Cristina Pilla Della Méa \\ Carlisa Peccin \\ Faculdade Meridional (IMED)
}

\begin{abstract}
Resumo
Objetivou-se avaliar sintomas de ansiedade, depressão e uso de substâncias psicoativas em pacientes que realizaram a cirurgia bariátrica. Trata-se de um estudo transversal, no qual foram avaliados 20 pacientes entre 1 a 2 anos pós-cirurgia. Utilizou-se um questionário sociodemográfico, Escalas Beck de Ansiedade (BAI) e de Depressão (BDI) e o Teste de Triagem do Envolvimento com Álcool, Cigarro e Outras Substâncias (ASSIST). Os resultados demonstraram que 80\% $(n=16)$ dos pacientes apresentaram sintomas de ansiedade leves, e 85\% ( $n=17)$ sintomas depressivos leves, e 15\% $(n=3)$ dos pacientes indicaram a necessidade de intervenção breve para uso de álcool. Houve correlação significativa entre os escores totais do ASSIST e do BDI. Conclui-se que a cirurgia bariátrica não é um fator significativo para desenvolvimento desses sintomas, porém destaca-se a importância de acompanhamento psicológico no pós-operatório.
\end{abstract}

Palavras-chave: obesidade, cirurgia bariátrica, depressão, ansiedade, uso de substâncias

\begin{abstract}
The objective of this study was to evaluate symptoms of anxiety, depression and psychoactive substance use in patients who underwent bariatric surgery. This was a cross-sectional study, in which 20 patients between 1 and 2 years after surgery were evaluated. The instruments used: sociodemographic questionnaire, Beck Anxiety (BAI) and Depression Inventory (BDI) and Alcohol, Smoking and Substance Involvement Screening Test (ASSIST). The results showed that $80 \%(n=16)$ of the patients presented mild anxiety symptoms and $85 \%(n=17)$ mild depressive symptoms and $15 \%(n=3)$ of the patients indicated the need for brief intervention for alcohol use. There was a significant correlation between the total ASSIST and BDI scores. It is concluded that bariatric surgery is not a significant factor for the development of these symptoms, but the importance of psychological monitoring in the postoperative period stands out.

Keywords: obesity, bariatric surgery, depression, anxiety, substance use

\section{Resumen}

El objetivo deste estudio fue evaluar los síntomas de ansiedad, depresión y abuso de sustancias en pacientes sometidos a cirugía bariátrica. Este es un estudio transversal en el que 20 pacientes estaban entre 1 y 2 años después de la cirugía. Se utilizó un cuestionario sociodemográfico, Escala de Ansiedad de Beck (EAB), Escala de Depresión de Beck (EDB) y el Teste de Triagem do Envolvimento com Álcool, Cigarro e Outras Substâncias (ASSIST). Los resultados mostraron que $80 \%(n=16)$ de los pacientes experimentaron síntomas de ansiedad leve, $85 \%(n=17)$ tuvieron síntomas depresivos leves y $15 \%(n=3)$ indicaron la necesidad de intervención breve para el uso de alcohol. Hubo correlación significativa entre el puntaje total del ASSIST y de la EDB. La conclusión del estudio es que la cirugía bariátrica no es un factor importante en el desarrollo de esos síntomas, pero es relevante el apoyo psicológico postoperatorio.

Palabras clave: obesidad, cirugía bariátrica, depresión, ansiedad, uso de sustancias
\end{abstract}

\footnotetext{
1 Endereço de contato: Faculdade Meridional (IMED). Rua Senador Pinheiro, 304, Passo Fundo, RS. CEP 99070-220. E-mail: cristina.mea@imed.edu.br
} 


\section{Introdução}

A obesidade é definida como o acúmulo excessivo de tecido adiposo e pelo excesso de consumo alimentar comparado com o consumo de energia para as atividades vitais, ou seja, um balanço energético positivo comprometendo a saúde e a qualidade de vida do indivíduo (Barbieri \& Mello, 2012). A obesidade é classificada segundo o Índice de Massa Corpóreo (IMC), dividindo o peso corporal em $\mathrm{kg}$ pelo quadrado da altura em $\mathrm{m}^{2}$, sendo considerada obesidade grau I quando o IMC está entre 30 a $34,9 \mathrm{~kg} / \mathrm{m}^{2}$, grau II é quando o IMC é entre 35 a 39,9kg/m² e grau III é quando o IMC é maior que $40 \mathrm{~kg} / \mathrm{m}^{2}$ (Associação Brasileira para o Estudo da Obesidade e da Síndrome Metabólica [ABESO], 2016). Em uma pesquisa realizada no Brasil, a prevalência de obesidade em adultos foi de 17\%, sendo maior em homens (18\%) quando comparada com mulheres (16,2\%) (Brasil, 2017).

O tratamento da obesidade é multidisciplinar, e busca-se modificar o estilo de vida. Envolve uma reeducação alimentar gradativa, atividade física regular e acompanhamento psicológico e, em alguns casos, uso de medicação. Quando não há resultados nesses tratamentos, indica-se a cirurgia bariátrica (Brasil, 2014; Oliveira \& Almeida, 2012).

O objetivo da cirurgia bariátrica não se restringe somente à perda de peso, mas inclui a meIhora no funcionamento psicossocial, na qualidade de vida e também para a estabilização dos parâmetros clínicos. Uma rigorosa avaliação é realizada antes de indicá-la, por profissionais das áreas: da medicina, psicologia, nutrição e enfermagem (Fandiño, Benchimol, Coutinho, \& Appolinário, 2004; Costa, Ivo, Cantero, \& Tognini, 2009; Gordon, Kaio, \& Sallet, 2011). Cabe destacar que, para a cirurgia ter sucesso, depende da motivação do paciente para uma reeducação alimentar e a mudança no estilo de vida (Fandiño et al., 2004; Gordon et al., 2011).

A portaria número 424, de 19 de março de 2013, do Ministério da Saúde define diretrizes para o tratamento cirúrgico da obesidade e apresenta as indicações e as contraindicações à realização da cirurgia bariátrica. As indicações contemplam: indivíduos maiores de 18 anos, com IMC $50 \mathrm{Kg} / \mathrm{m}^{2}$; indivíduos com IMC $40 \mathrm{Kg} / \mathrm{m}^{2}$ (com ou sem comorbidades), sem sucesso no tratamento clínico, por no mínimo dois anos e que tenham seguido protocolos clínicos; indivíduos com IMC > $35 \mathrm{~kg} / \mathrm{m} 2$ com comorbidades, tais como: risco cardiovascular, diabetes mellitus e/ou hipertensão arterial sistêmica de difícil controle, apneia do sono, doenças articulares degenerativas, sem sucesso no tratamento clínico realizado por no mínimo dois anos. Ainda, o paciente não deve utilizar nenhuma droga ilícita ou ser dependente de álcool, não deve apresentar quadro psicótico ou demenciais (graves ou moderados). Também se faz necessário que tanto o paciente quanto seus familiares estejam cientes das mudanças de hábitos e que o paciente precisa de acompanhamento pós-operatório com a equipe multidisciplinar, a longo prazo (Conselho Federal de Medicina [CFM], 2010; Brasil, 2013).

As mudanças psicológicas decorrentes da cirurgia bariátrica são marcantes. Em alguns casos, há expectativas além do emagrecimento, como por exemplo, a resolução dos conflitos interpessoais e conjugais, problemas emocionais, sociais e profissionais, assim como mudanças de traços de suas personalidades. Os pacientes imaginam que vão se resolver por terem se libertado da obesidade, o que acaba levando a uma possível frustração diante de desejos não conquistados pela cirurgia (Leal \& Baldin, 2007; Ehrenbrink, Pinto, \& Prando, 2009).

Estima-se que de $20 \%$ a $70 \%$ dos pacientes obesos que procuram tratamento tem histórico de algum transtorno mental, tendo uma prevalência de $22 \%$ para transtornos do humor 
e 18,1\% de transtornos ansiosos (Amber, Cox, Murray, \& Sareen, 2008; Pull, 2010). Esses pacientes merecem maior atenção por parte dos profissionais no momento da avaliação pré-operatória e demandam intervenções eficazes antes, durante e após a cirurgia bariátrica, pois diante das restrições alimentares no pós-operatório, pode haver uma resposta mal adaptativa do indivíduo (Dobrow, Kamenetz, \& Devlin, 2002; Song \& Fernstrom, 2008).

Após um ano ou um pouco mais da realização da cirurgia, se não houver um acompanhamento terapêutico satisfatório, o indivíduo com obesidade pode buscar novas formas de expressão. Observam-se duas vias que podem se formar: a depressiva e a da compulsão. A primeira começa com sintomas vagos: sensação de vazio, perda de interesse por coisas que traziam prazer, perda de rendimento no trabalho e angústia. A segunda refere-se ao traço impulsivo, existente na personalidade do obeso, no qual o indivíduo vai em direção ao alimento. Através do consumo de bolachas que dissolvem na boca, pelo biscoito de polvilho, leite condensado, sorvete e chocolate. Há um descuido, e o peso começa a avançar através do consumo novamente (Magdaleno Jr., Chaim, \& Turato, 2009). Em pesquisa realizada com 47 pacientes submetidos à cirurgia bariátrica com o método baypass gástrico, 31 pacientes (65,95\%) apresentaram sintomas psíquicos como: dependência afetiva, ansiedade e angústia, imagem corporal distorcida, transtornos compulsivos obsessivos e depressão, segundo avaliação psicológica e psiquiátrica (Pull, 2010; Dellosso, Silva, \& Cunha, 2013).

A ansiedade também é considerada como contribuinte para o aumento de peso e prejudicial no pós-operatório, algumas pesquisas apontam para maiores prevalências desse sintoma do que depressivos. Em um estudo, foram encontrados níveis mais altos de ansiedade após 6 meses de cirurgia com 14\% da amostra (50 pacientes), enquanto que 8\% dos sujeitos apresentaram sintomas depressivos (Porcu, Franzin, Abreu, Previdelli, \& Astolfi, 2011). Nesse sentido, Cavalcante (2009) acrescenta que os pacientes pós-cirúrgicos apresentam mudanças em relação à alimentação, ou seja, a diminuição e modificação dos hábitos alimentares, e isso pode provocar aumento nos sintomas de ansiedade.

Outro ponto a se destacar no pós-operatório é o uso/abuso de substâncias psicoativas. A prevalência de transtornos relacionados ao uso de álcool em pacientes no pré-operatório e pós-operatório, dois anos após a cirurgia, aumentou segundo King et al. (2012). Os dados foram associados ao sexo masculino e idade mais jovem, usuários de tabaco, que consumiam regularmente álcool e drogas. Diante do exposto, o paciente pode, através da bebida alcoólica, tentar satisfazer a vontade de comer que ele tinha antes da cirurgia (Song \& Fernstrom, 2008; Barros, Moreira, Frota, \& Caetano, 2013).

O presente estudo teve como objetivo avaliar sintomas de ansiedade, depressão e uso de substâncias psicoativas em pacientes entre 1 a 2 anos da realização da cirurgia bariátrica.

\section{Método}

\section{Delineamento}

Trata-se de um estudo observacional transversal, com variáveis de desfecho quantitativas. O estudo transversal é recomendado quando o objetivo é de estimar a frequência com que um determinado evento de saúde se manifesta em uma população específica, além dos fatores ou variáveis associadas com este (Marconi \& Lakatos, 2011). 


\section{Participantes}

A amostra foi não probabilística, por conveniência. Os critérios de inclusão dos sujeitos na pesquisa foram: indivíduos maiores de 18 anos; homens e mulheres; ter realizado a cirurgia bariátrica entre 1 a 2 anos e realizar consultas de acompanhamento com a equipe multidisciplinar. Como critérios de exclusão: história anterior à cirurgia de abuso ou dependência de substância psicoativa; diagnóstico anterior à cirurgia de transtorno de humor ou transtorno de ansiedade; pacientes que estão em processo de luto ou que passaram por uma perda significativa (como separação conjugal ou perda de emprego) e pacientes que não sabiam ler nem escrever.

Primeiramente foi realizada uma entrevista de investigação dos critérios de inclusão e exclusão da amostra. Foram abordados 37 sujeitos que realizaram cirurgia bariátrica no momento em que retornavam para consulta médica de rotina, porém 17 pacientes foram excluídos da pesquisa, $27 \%(n=10)$ por apresentarem diagnóstico de transtorno de humor, 10,8\% $(n=4)$ com dependência de substância psicoativa (tabaco), 5,4\% em situações de luto ( $n=2$ ) e $2,7 \%(n=1)$ havia sofrido um acidente recentemente. Assim, o presente estudo se deu com 20 sujeitos que realizaram cirurgia bariátrica entre 1 a 2 anos.

\section{Instrumentos}

Foram aplicados os seguintes instrumentos:

a) Questionário sociodemográfico: construído para este estudo, composto de 9 questões, com a finalidade de levantar os dados sociodemográficos da amostra.

b) Inventário Beck de Ansiedade (BAI): escala composta por 21 itens que tem como objetivo avaliar a intensidade de sintomas de ansiedade. A soma dos escores tem como resultado sintomas de ansiedade em nível mínimo, leve, moderado e grave (Cunha, 2001).

b) Inventário de Depressão de Beck (BDI): escala composta de 21 questões que tem o objetivo de avaliar a intensidade de sintomas depressivos. A soma dos escores tem como resultado sintomas depressivos em nível mínimo, leve, moderado e grave (Cunha, 2001).

c) Teste de Triagem do Envolvimento com Álcool, Cigarro e Outras Substâncias (ASSIST): questionário estruturado com oito questões, que tem o objetivo de rastrear o uso de substâncias psicoativas, que abrange: tabaco, álcool, maconha, cocaína, estimulantes, sedativos, inalantes, alucinógenos e opiáceos. A pontuação classifica quanto ao risco de problemas ou dependência em função do uso da substância (baixo risco, risco moderado e alto risco) (Henrique, De Micheli, Lacerda, Lacerda, \& Formigoni, 2004).

\section{Procedimentos}

Após aprovação do Comitê de Ética (CEP), número 481.778, realizou-se contato com uma Clínica Particular de Gastrologia que realiza cirurgias bariátricas no interior do estado do Rio Grande do Sul, a fim de apresentar a proposta da pesquisa. O médico responsável autorizou o estudo, e a psicóloga do local acompanhou a execução da pesquisa.

A coleta foi feita individualmente numa sala nas dependências da Clínica de Gastrologia. Aos participantes, esclareceram-se aspectos relacionados à natureza e os propósitos do estudo, bem como da responsabilidade do pesquisador quanto ao sigilo da identidade dos 
envolvidos. Foram sanadas possíveis dúvidas e, em seguida, lido e assinado o Termo de Consentimento Livre e Esclarecido (TCLE), de acordo com a Resolução 466/12 do Conselho Nacional de Saúde.

\section{Análise dos Dados}

Os dados foram analisados e levantados quantitativamente, com descrição e interpretação dos resultados através do Microsoft Excel ${ }^{\circledR}$. Efetuaram-se comparações entre as variáveis e correlação de Pearson entre os sintomas encontrados com a cirurgia bariátrica, comparando-se os resultados com pesquisas semelhantes.

\section{Resultados e Discussão}

Conforme indicado pela Tabela 1, a maioria dos participantes do presente estudo era do gênero feminino 55\% $(n=11)$, a predominância de mulheres é habitual na demanda aos serviços de cirurgia da obesidade como aponta pesquisas de Cavalcante (2009) e Porcu et al. (2011), Almeida, Zanatta e Rezende (2012), que pontuam a exigência social de um corpo feminino magro em função de questões culturais. Na escolaridade predominou nível de Ensino Médio 45\% (n=9), esse dado está de acordo com outras pesquisas (Oliveira \& Yoshida, 2009; Porcu et al., 2011).

A maioria dos pacientes é casada $70 \%(n=14)$, o que corrobora pesquisas semelhantes (Oliveira \& Yoshida, 2009; Porcu et al., 2011; Mota, 2012). A prevalência de pacientes casados, ou com união estável, é um fator positivo e sugere vínculos que atuam como suporte ao paciente no pós-operatório (Monteiro \& Santos, 2011). A idade média dos indivíduos da amostra foi de 44,5 anos (DP=12,47), com o mínimo de 27 e máximo de 66 anos, o que coincide com outras pesquisas que identificaram a média de 44 anos (Porcu et al., 2011), de 40 anos (Rojas, Brante, Miranda, \& Pérez-Luco, 2011) e de 42 anos (Mota, 2012).

Em relação ao trabalho, estão atualmente desempenhando atividades profissionais $85 \%$ $(n=17)$, verificou-se a prevalência de determinadas áreas de atuação, como a área administrativa $20 \%(n=4)$ e comércio/vendas $20 \%(n=4)$. As profissões da área administrativa envolvem empresários, bancários e funcionários públicos, principalmente, ao passo que a área comércio/vendas representa as profissões de comerciante e vendedores. 


\section{Tabela 1}

Caracterização geral da amostra

\begin{tabular}{llcc}
\hline Questionário sociodemográfico & \multicolumn{1}{c}{ Item } & N & \% \\
\hline \multirow{2}{*}{ Gênero } & Feminino & 11 & 55 \\
& Masculino & 9 & 45 \\
Escolaridade & Ensino fundamental & 4 & 20 \\
& Ensino médio & 9 & 45 \\
& Ensino superior & 7 & 35 \\
Estado civil & Solteiro (a) & 1 & 5 \\
& Casado (a) & 14 & 70 \\
Trabalha atualmente & Separado (a) & 2 & 10 \\
& União estável & 3 & 15 \\
& Sim & 17 & 85 \\
& Não & 3 & 15 \\
& Administrativo & 4 & 20 \\
Área de atuação & Agricultura & 2 & 10 \\
& Aposentado / pensionista & 2 & 10 \\
& Comércio / vendas & 4 & 20 \\
& Do lar & 1 & 5 \\
& Educação & 2 & 10 \\
& Operacional & 2 & 10 \\
& Religião & 1 & 5 \\
& Saúde & 2 & 10 \\
\hline
\end{tabular}

No que se refere às questões da cirurgia bariátrica, o tempo médio de realização desse procedimento nos pacientes avaliados foi de 17,55 meses ( $D P=4,96)$. A análise descritiva indicou que a maioria dos pacientes avaliados despendeu mais de 5 anos da vida em tentativas para perder peso antes da cirurgia $75 \%(n=15)$, de acordo com as informações indicadas na Tabela 2. Aproximadamente $40 \%(n=8)$ dos pacientes relataram terem perdido entre 31 e 40 kg no total, e, inclusive, grande parte dos pacientes demonstra-se satisfeita com o seu peso atual 90\% (n=18). Um dado interessante é que nenhum paciente tem acompanhamento psicológico ou psiquiátrico atualmente, exceto as consultas periódicas com um psicológico oferecidas pela equipe multidisciplinar durante o período de um ano pós-cirurgia.

\section{Tabela 2}

Análise descritiva de variáveis relacionadas ao peso e a realização de acompanhamento após a cirurgia bariátrica

\begin{tabular}{lccc}
\hline \multicolumn{1}{c}{ Questões ligadas à cirurgia } & Item & $\mathbf{N}$ & \% \\
\hline Tempo antes da cirurgia que realizou & 01 a 03 anos & 4 & 20 \\
tentativas de perder peso & 03 a 05 anos & 1 & 5 \\
& Mais de 05 anos & 15 & 75 \\
Está satisfeito com o seu peso atual & Sim & 18 & 90 \\
& Não & 2 & 10 \\
Peso diminuído após cirurgia & 21 a 30 kg & 5 & 25 \\
& 31 a 40 kg & 8 & 40 \\
Realiza tratamento psicológico & Mais de 41 kg & 7 & 35 \\
Ou psiquiátrico? & Sim & 0 & 0 \\
\hline
\end{tabular}


As análises dos instrumentos que avaliam sintomas de ansiedade (BAI), depressão (BDI) e uso de substâncias podem ser visualizadas na Tabela 3. Referente aos escores do BAI observou-se que $80 \%(n=16)$ dos pacientes avaliados indicavam a presença de sintomas mínimos de ansiedade, 15\% ( $n=3)$ pontuavam sintomas leves e $5 \%(n=1)$ sintomas moderados. Nenhum dos pacientes avaliados demonstrou a presença de sintomas graves de ansiedade. O escore médio total observado no BAl foi de 5,70 ( $D P=5,921)$, indicando a presença de sintomas com intensidade mínima de ansiedade.

No que diz respeito aos escores do BDI, verificou-se que 85\% $(n=17)$ dos pacientes apresentavam sintomas depressivos de intensidade mínima, seguidos por sintomas de intensidade leve $10 \%(n=2)$ e moderada $5 \%(n=1)$. Nenhum dos pacientes avaliados pontuou sintomas de intensidade grave, de forma que a média total dos sintomas apresentados pelo BDI foi de 4,70 (DP=4,28), sugerindo a presença de sintomas mínimos ou ausentes de depressão. Ainda, a análise dos escores do BDI e do BAI para pacientes após a cirurgia bariátrica não confirmou a presença de uma correlação significativa entre esses instrumentos $(r=0,065$, $p=0,05)$. Ainda, a análise da correlação entre os instrumentos indicou somente correlação significativa entre os escores totais do BDI e do ASSIST $(r=0,729, p=0,01)$, sugerindo que, nos casos de pacientes após cirurgia bariátrica, a intensidade dos sintomas depressivos aumenta proporcionalmente ao uso de substâncias, ou vice-versa.

\section{Tabela 3}

Caracterização da intensidade de sintomas ansiedade, depressão e uso de substâncias em pacientes após a realização da cirurgia bariátrica

\begin{tabular}{|c|c|c|c|c|c|c|c|}
\hline \multirow{2}{*}{ Instrumento } & & \multicolumn{2}{|c|}{ Amostra total } & \multicolumn{2}{|c|}{ Sexo Masculino ( $\mathrm{N}=9$ ) } & \multicolumn{2}{|c|}{ Sexo Feminino( $\mathrm{N}=11)$} \\
\hline & & Média & DP & Média & DP & Média & DP \\
\hline $\mathrm{BAl}$ & Ansiedade & 4.70 & 4.28 & 2.33 & 2.44 & 6.63 & 4.57 \\
\hline \multirow[t]{3}{*}{ BDI } & Depressão & 5.70 & 5.921 & 3.44 & 3.47 & 7.54 & 6.97 \\
\hline & Tabaco & 0.41 & 1.53 & 0.00 & 0.00 & 0.64 & 2.11 \\
\hline & Álcool & 4.09 & 4.79 & 4.00 & 3.96 & 4.09 & 5.96 \\
\hline \multirow[t]{3}{*}{ ASSIST } & Maconha & 0.32 & 1.28 & 0.67 & 2.00 & 0.00 & 0.00 \\
\hline & Cocaína & 0.95 & 0.89 & 0.89 & 0.93 & 1.00 & 1.00 \\
\hline & Escore total & 5.65 & 6.68 & 5.56 & 4.64 & 5.72 & 6.65 \\
\hline
\end{tabular}

Os achados nesta pesquisa são corroborados por outro estudo que mostrou que 6,06\% dos pacientes apresentaram sintomas de ansiedade em nível moderado/grave e 3\% dos pacientes apresentaram sintomas depressivos de intensidade entre 6 a 33 meses pós-cirurgia (Oliveira \& Yoshida, 2009). Ainda, Almeida et al. (2012) encontraram sintomas mínimos de ansiedade e depressivos no período de 12 meses após a realização do procedimento. Em uma avaliação pós-operatória (entre 6 a 12 meses) com 31 mulheres, percebeu-se uma meIhora nos sintomas de ansiedade e depressivos (Zaldívar, Horcajadas, Martínez, \& Romero, 2009; Barroqueiro, Araújo, Barroqueiro, Araújo, \& Garcia, 2012). Nesse sentido, Andersen et al. (2010) e Mota (2012) também avaliaram os sintomas antes e após a cirurgia e referem melhora nos sintomas de ansiedade e depressivos, sugerindo que o procedimento auxilia nesses sintomas, reduzindo-os. 
Pode-se inferir, através desses dados, que a cirurgia bariátrica não é um fator significativo que contribui para desenvolvimento de sintomas de ansiedade e depressivos, porém todos os pacientes foram obrigatoriamente acompanhados no período de até 12 meses pós-cirúrgico pela psicóloga da equipe, como prevê portaria do Ministério da Saúde (Brasil, 2013), o que pode ser um fator protetivo dos sujeitos. Entende-se na literatura como de fundamental importância o acompanhamento psicológico e nutricional até no mínimo 24 meses após a realização da cirurgia (Ehrenbrink et al., 2009; Oliveira \& Almeida, 2012; Barros et al., 2013). Em uma pesquisa com 15 pacientes pós 12 a 20 meses de cirurgia, conclui-se que 33,33\% apresentaram sintomas depressivos leves; $6,67 \%$, moderados e $6,67 \%$ com sintomas graves. Quanto aos sintomas de ansiedade, 26,6\%, apresentaram sintomas leves, 13,33\%, com ansiedade moderada e 13,33\%, com ansiedade grave (Cavalcante, 2009).

Quanto à diferença entre os gêneros, também apresentada na Tabela 3, é possível observar a presença de maiores escores de sintomas de ansiedade e depressivos nas pacientes do sexo feminino, quando comparadas aos homens, sugerindo que sintomas depressivos e ansiosos são mais frequentes nas mulheres. Esse dado é evidenciado pela literatura, na qual se afirma que há um predomínio de mulheres sobre os homens com transtornos de ansiedade e depressão (American Psychiatric Association [APA], 2013).

Em relação aos escores ASSIST, verificou-se que 75\% ( $n=15)$ dos indivíduos não apontavam necessidade de nenhuma intervenção, ao passo que $15 \%(n=3)$ indicavam a necessidade de intervenção breve para o uso de álcool, $5 \%(n=1)$ manifestavam a necessidade de intervenção breve para o uso de tabaco, e 5\% $(n=1)$ necessitavam de intervenção breve para o uso de maconha. A correlação de Pearson, efetuada a 5\% de probabilidade, também indicou uma correlação significativa entre o tempo de realização da cirurgia bariátrica e os escores totais do ASSIST ( $r=0,809, p=0,0034)$, o que sugere que os escores associados ao uso de substâncias aumentam conforme o tempo de realização da cirurgia.

Esse dado torna-se importante no sentido de que, em outras pesquisas, já apontam o desenvolvimento do uso de álcool com maior frequência após a cirurgia bariátrica, como verificaram mais recentemente King et al. (2012) e Conason et al. (2013), em que, após 24 meses da cirurgia, houve um aumento no consumo de álcool e tabaco. Marchesini (2010) avaliou uma amostra de 43 pacientes, e 11\% relataram substituir a comida pela bebida.

Diante dos resultados obtidos através do estudo, estes foram repassados à profissional psicóloga responsável pela instituição de saúde para que ela possa realizar os devidos encaminhamentos aos pacientes que apresentaram sintomas de ansiedade, depressão e uso de substâncias psicoativas.

\section{Considerações Finais}

O objetivo do estudo foi avaliar sintomas de ansiedade, depressão e uso de substâncias psicoativas após cirurgia bariátrica, por entender que esse procedimento causa grandes mudanças corporais e também no estilo de vida, pois o paciente precisa adotar uma série de cuidados para manter o peso a longo prazo.

Os resultados indicam que os pacientes não apresentaram sintomas de ansiedade e depressão significativos, nenhum paciente apresentou sintomas graves, apenas leve e moderado em menor prevalência. Já o uso de álcool parece ser mais comum, o que pode ser enten- 
dido como uma estratégia de compensação encontrada pelos indivíduos, pois não podendo se alimentar como antes, podem encontrar na bebida uma forma de prazer semelhante. Um dado importante é a correlação positiva entre depressão e uso de álcool, na medida em que os sintomas de depressão ainda que mínimos aumentam, o consumo de álcool também se eleva, ou vice-versa. Este estudo também mostrou que existe uma correlação entre o tempo de cirurgia bariátrica e o uso de álcool e outras substâncias, mesmo não sendo evidenciados escores graves que necessitem de intervenções.

Portanto indica-se o acompanhamento psicológico para pacientes que se submeteram à cirurgia bariátrica para prevenção no desenvolvimento desses sintomas, aceitação do seu corpo, para auxiliar na manutenção do peso e mudança do seu estilo de vida, pois, mesmo esses sintomas não sendo classificados como graves, a cirurgia se constitui em um marco na vida do sujeito e, dependendo de como ele reage a fatores de mudanças, poderá influenciar na sua evolução no pós-cirúrgico, considerando-se que na pesquisa nenhum paciente realizava psicoterapia.

Embora se tenha constatado que a cirurgia bariátrica não é um fator significativo para o desenvolvimento desses sintomas, sugere-se a necessidade de novos estudos. Uma das limitações da pesquisa é o tamanho da amostra, sugerindo avaliar os sintomas com um maior número de participantes. Além disso, relacionar sintomas depressivos e o consumo de álcool nesses pacientes poderá indicar com maior precisão se a correlação encontrada na pesquisa se mantém através do tempo ou varia de acordo com a intensidade dos sintomas apresentados.

\section{Referências}

Almeida, S. S., Zanatta, D. P., \& Rezende, F. F. (2012). Imagem corporal, ansiedade e depressão em pacientes obesos submetidos a cirurgia bariátrica. Estudos de Psicologia, 17(1), 153-160. doi: 10.1590/S1413-294X2012000100019

Amber A. M., Cox, B. J., Murray, W. E., \& Sareen, J. (2008). Associations between body weight and personality disorders in a nationally representative sample. Psychosomatic Medicine, 70(1), 1012-1019. doi: 10.1097/PSY.0b013e318189a930

American Psychiatric Association. (2013). Diagnostic and statistical manual of mental disorders: DSM-5 (5th ed.). Arlington, VA: American Psychiatric Publishing.

Andersen, J. R., Aasprang, A., Bergsholm, P., Sletteskog, N., Vage V., \& Natvig, G. K. (2010). Anxiety and depression in association with morbid obesity: changes with improved physical health after duodenal switch. Health and Quality of Life Outcomes, 8(1), 8-52. doi: 10.1186/1477-7525-8-52

Associação Brasileira para o Estudo da Obesidade e da Síndrome Metabólica. (2016). Diretrizes brasileiras de obesidade 2016 (4a ed.). São Paulo, SP. Disponível em http:// www.abeso.org.br/uploads/downloads/92/57fccc403e5da.pdf

Barbieri, A. F. \& Mello, R. A. (2012). As causas da obesidade: uma análise sob a perspectiva materialista histórica. Revista da Faculdade de Educação Física, 10(1), 133-153. Disponível em https://periodicos.sbu.unicamp.br/ojs/index.php/conexoes/article/view/8637693/5384

Barroqueiro, R. S. B., Araújo, F. L. S. M., Barroqueiro, E. S. B., Araújo, G. F., \& Garcia, J. B. S. (2012). Função sexual feminina, sintomas de ansiedade e depressão em mulheres após 
cirurgia bariátrica. Revista de Ciências da Saúde, 14(1), 60-67. Disponível em http://www. periodicoseletronicos.ufma.br/index.php/rcisaude/article/view/1284

Barros, L. M., Moreira, R. A. N., Frota, N. M., \& Caetano, J. A. (2013). Mudanças na qualidade de vida após a cirurgia bariátrica. Revista de Enfermagem UFPE Online, 7(5), 1365-1375. doi: 10.5205/reuol.3960-31424-1-SM.0705201315

Brasil. (2013). Portaria n. 424/GM, 19 de março de 2013. Redefine as diretrizes para a organização da prevenção e do tratamento do sobrepeso e obesidade como linha de cuidado prioritária da Rede de Atenção à Saúde das Pessoas com Doenças Crônicas. Disponível em http://www.poderesaude.com.br/novosite/images/stories/Publicaes_20.03.2013_-_Ill.pdf

Brasil. (2014). Estratégias para o cuidado da pessoa com doença crônica: Obesidade. Brasília, DF: Ministério da Saúde. Disponível em http://bvsms.saude.gov.br/bvs/publicacoes/estrategias_cuidado_doenca_cronica_obesidade_cab38.pdf

Brasil. (2017). Vigitel Brasil 2015 Saúde Suplementar: Vigilância de fatores de risco e proteção para doenças crônicas por inquérito telefônico[recurso eletrônico]. Brasília, DF: Ministério da Saúde. Disponível em_http://www.ans.gov.br/images/stories/Materiais_para_pesquisa/Materiais_por_assunto/2015_vigitel.pdf

Cavalcante, R. C. (2009). Análise comportamental de obesos mórbidos e de pacientes submetidos à cirurgia bariátrica (Dissertação de Mestrado, Universidade Federal de Pernambuco, Recife). Disponível em http://repositorio.ufpe.br/handle/123456789/8655

Conason, A., Teixeira, J., Hsu, C. C., Puma L., Knafo, D., \& Geliebter, A. (2013). Substance use following bariatric weight loss surgery. Jama Surgery, 148(2), 145-150. doi: 10.1001/2013. jamasurg.265

Conselho Federal de Medicina. (2010). Resolução n. 1.942, 12 de fevereiro de 2010. Altera a Resolução CFM n. 1.766, que estabelece normas seguras para o tratamento cirúrgico da obesidade mórbida, definindo indicações, procedimentos e equipe. Disponível em http:// arquivos.cremesc.org.br/publicacao/Revista\%20112\%202010/Revista_112_2010/files/ assets/downloads/page0015.pdf

Costa, A. C. C., Ivo, M. L., Cantero, W. B., \& Tognini, J. R. F. (2009). Obesidade em pacientes candidatos a cirurgia bariátrica. Acta Paul Enfermagem, 22(1), 55-59. doi: 10.1590/ S0103-2100200900010000

Cunha, J. A. (2001). Manual da versão em português das Escalas Beck. São Paulo: Casa do Psicólogo.

Dellosso, A. C. A., Silva, M. F. F., \& Cunha, M. C. (2013). Aspectos orgânicos, psíquicos e nutricionais em pacientes bariátricos. Distúrbios da comunicação, 25(2), 277-283. Disponível em https://revistas.pucsp.br//index.php/dic/article/view/12721

Dobrow, I. J., Kamenetz, C., \& Devlin, M. J. (2002). Aspectos psiquiátricos da obesidade. Revista Brasileira de Psiquiatria, 24(3), 63-67. Disponível em http://www.scielo.br/pdf/\%0D/rbp/v24s3/13975.pdf

Ehrenbrink, P. P., Pinto, E. P., \& Prando, F. L. (2009). Um novo olhar sobre a cirurgia bariátrica e os transtornos alimentares. Psicologia Hospitalar, 7(1), 88-105. Disponível em http:// pepsic.bvsalud.org/pdf/ph/v7n1/v7n1a06.pdf

Fandiño, J., Benchimol, A. K., Coutinho, W. F., \& Appolinário, J. C. (2004). Cirurgia bariátrica: Aspectos clínico-cirúrgicos e psiquiátricos. Revista de Psiquiatria, 26(1), 47-51. Disponível em http://www.scielo.br/pdf/rprs/v26n1/20476.pdf 
Gordon, P. C., Kaio, G. H., \& Sallet, P. C. (2011). Aspectos do acompanhamento psiquiátrico de pacientes obesos sob tratamento bariátrico: Revisão. Revista de Psiquiatria Clínica, 38(4), 148-154. doi: 10.1590/S0101-60832011000400007

Henrique, I. F. S., De Micheli, D., Lacerda, R. B., Lacerda, L. A., \& Formigoni, M. L. O. S. (2004). Validação da versão brasileira do teste de triagem do envolvimento com álcool, cigarro e outras substâncias (ASSIST). Revista Associação Médica Brasileira, 50(2), 199-206. doi: 10.1590/S0104-42302004000200039

King, W. C., Chen, J. J., Mitchell, J. E., Kalarchian, M. A., Steffen, K. J., Engel, S. G., C., Courcoulas, A. P., Pories, W. J., \& Yanovski, S. Z. (2012). Prevalence of alcohol use disorders before and after bariatric surgery. The Journal of the American Medical Association (JAMA), 307(23), 2516-2525.doi: 10.1001/jama.2012.6147

Leal, C. W., \& Baldin, N. (2007). O impacto emocional da cirurgia bariátrica em pacientes com obesidade mórbida. Revista de Psiquiatria do Rio Grande do Sul, 29(3), 324-327. doi: 10.1590/S0101-81082007000300013

Magdaleno Jr., R., Chaim, E. A., \& Turato, E. R. (2009). Características psicológicas de pacientes submetidos à cirurgia bariátrica. Revista de Psiquiatria do Rio Grande do Sul, 31(1), 73-78. doi: 10.1590/S0101-81082009000100013

Marchesini, S. D. (2010). Acompanhamento psicológico tardio em pacientes submetidos à cirurgia bariátrica. ABCD: Arquivos Brasileiros de Cirurgia Digestiva, 23(2), 108-113. Disponível em http://www.scielo.br/pdf/abcd/v23n2/10.pdf

Marconi, M. A., \& Lakatos, E. M. (2011). Metodologia científica (6a ed.). São Paulo: Atlas.

Monteiro, M. S. S., \& Santos, G. S. (2011). Prevalência de transtorno de ansiedade e depressão em pacientes candidatos a cirurgia bariátrica. Revista Interdisciplinar NOVAFAPI, 4(1), 19-24. Disponível em http://uninovafapi.edu.br/sistemas/revistainterdisciplinar/v4n1/ pesquisa/p3_v4n1.pdf

Mota, D. C. L. (2012). Comportamento alimentar, ansiedade, depressão e imagem corporal em mulheres submetidas à cirurgia bariátrica (Dissertação de Mestrado, Programa de Pós-Graduação em Psicobiologia, Universidade de São Paulo). Disponível em http://www. teses.usp.br/teses/disponiveis/59/59134/tde-11062012-165343/pt-br.php

Oliveira, J. H. A., \& Yoshida, E. M. P. (2009). Avaliação psicológica de obesos grau III antes e depois de cirurgia bariátrica. Psicologia: Reflexão e Crítica, 22(1), 12-19. doi: 10.1590/ S0102-79722009000100003

Oliveira, L. H., \& Almeida, P. (2012). Obesidade: Aspectos gerais dos fatores, tratamento e prevenção. Revista Polidisciplinar Eletrônica da Faculdade de Guairacá, 4(2), 34-46. Disponível em http://www.revistavoos.com.br/seer/index.php/voos/article/ view/224/03_Vol4.2_VOOS2012_CL

Porcu, M., Franzin, R., Abreu, P. B., Previdelli, I. T. S., \& Astolfi, M. (2011). Prevalência de transtornos depressivos e de ansiedade em pacientes obesos submetidos à cirurgia bariátrica. Acta Scientiarum, 33(2), 165-171. doi: 10.4025/actascihealthsci.v33i2.7653

Pull, C. B. (2010). Current psychological assessment practices in obesity surgery programs: what to assess and why. Current Opinion in Psychiatry, 23(1), 30-36. doi: 10.1097/ YCO.0b013e328334c817

Rojas, C., Brante, M., Miranda, E., \& Pérez-Luco, R. (2011). Descripción de manifestaciones ansiosas, depresivas y auto concepto en pacientes obesos mórbidos, some- 
tidos a cirugía bariátrica. Revista Médica de Chile, 139(5), 571-578. doi: 10.4067/ S0034-98872011000500002

Song, A., \& Fernstrom, M. H. (2008). Nutritional and psychological considerations after bariatric surgery. Aesthetic Surgery Journal, 28(2), 195-199. doi: 10.1016/j.asj.2008.01.005.

Zaldívar, S. S., Horcajadas, F. A., Martinez, J. J. G., \& Romero, S. S. (2009). Evolución de alteraciones psicopatológicas en pacientes con obesidade mórbida tras cirurgia bariátrica. Medicina Clínica, 133(6), 206-212. doi: 10.1016/j.medcli.2008.11.045

Recebido: 22/12/2014

Última revisão: 06/06/2017

Aceite final: 12/06/2017

\section{Sobre as autoras:}

Cristina Pilla Della Méa: Psicóloga. Docente do Curso de Psicologia da Faculdade Meridional (IMED). Mestre em Envelhecimento Humano (UPF), Especialista em Psicologia Clínica (IMED) e Especialista em Psicoterapias Cognitivo-Comportamentais (UNISINOS, São Leopoldo, RS). E-mail: cristina.mea@imed.edu.br

Carlisa Peccin: Psicóloga formada pela Faculdade Meridional (IMED). E-mail: carlisa_peccin@hotmail.com 